\title{
Offline optimization of wavelength allocation and laser power in nanophotonic interconnects
}

\author{
Jiating Luo ${ }^{1}$, Cedric Killian ${ }^{1}$, Sebastien Le Beux ${ }^{2}$, Daniel Chillet ${ }^{1}$, \\ Olivier Sentieys ${ }^{1}$, and Ian O'Connor ${ }^{2}$ \\ ${ }^{1}$ Univ Rennes, Inria, CNRS, IRISA, F-22305 Lannion, France \\ ${ }^{2}$ Ecole Centrale de Lyon, INL, Ecully, F-69134, France
}

December 10, 2018

\begin{abstract}
Optical Network-on-Chip (ONoC) is a promising communication medium for large-scale Multiprocessor System on Chip (MPSoC). Indeed ONoC can outperform classical electrical NoC in terms of energy efficiency and bandwidth density, in particular, because this medium can support multiple transactions at the same time on different wavelengths by using Wavelength Division Multiplexing (WDM). However, multiple signals sharing simultaneously the same part of a waveguide can lead to inter-channel crosstalk noise. This problem impacts the Signal to Noise Ratio (SNR) of the optical signals, which leads to an increase in the Bit Error Rate (BER) at the receiver side. If a specific BER is targeted, an increase of laser power should be necessary to satisfy the SNR. In this context, an important issue is to evaluate the laser power needed to satisfy the various desired communication bandwidths based on the BER performance requirements. In this paper, we propose an off-line approach that concurrently optimizes the laser power scaling and execution time of a global application. A set of different levels of power is introduced for each laser, to ensure that optical signal can be emitted with just-enough power to ensure targeted BER. As result, most promising solutions are highlighted for mapping a defined application onto 16-core ring-based WDM ONoC.
\end{abstract}

\section{Introduction}

Multiprocessor System-on-Chip (MPSoC) are evolving towards the integration of hundreds of cores on a single chip. Designing an efficient interconnect for such complex architectures is challenging due to the ever growing data exchange between processors. Networks on Chip (NoCs) have been proposed to overcome these issues but they are now reaching their performance limits. Indeed, packetization and depacketization of data significantly impact NoC latency [1] and 
the same bandwidth for all router ports is usually assumed while processors bandwidth requirements vary significantly [2]. As reported in [3], NoC switching activity accounts for over $50 \%$ of the interconnect power consumption. NoC efficiency thus needs to be further improved in order to efficiently interconnect systems with still increasing number of cores and required bandwidth density [4].

Nanophotonic interconnects is a promising technology to replace traditional electrical NoC. Indeed, Wavelength Division Multiplexing (WDM) allows the propagation of multiple signals simultaneously on a same waveguide [5], thus leading to high aggregated bandwidth. However, WDM also leads to interchannel crosstalk noise [6], which negatively impacts the Signal to Noise Ratio (SNR) and, therefore, the Bit Error Rate (BER). The BER to be reached depends on the application requirements and is closely related to the optical devices characteristics: it depends on the photodetector sensitivity [7], on the Microring Resonator transmission spectrum, and on the optical signal power emitted by the laser sources. The higher the number of signals propagating simultaneously, the higher the crosstalk and the higher the laser output power needed. This thus leads to the following conflicting objectives: high performance communications tend to rely on an exhaustive use of the available wavelengths while energy efficient communications involve a parsimonious use of signals which occupy distinct and separate wavelengths. Using an Optical NoC $(\mathrm{ONoC})$ to execute a given application is thus a tedious task, especially if performance, power and BER objectives are likely to evolve with the execution context.

This paper addresses this problem by proposing an off-line methodology allowing the exploration of performances and energy consumption trade-off for ONoC-based MPSoC. In order to find the best trade-off, we combine, for the first time, Wavelength Allocation (WA) and laser power scaling. The key ideas are to i) allocate just enough wavelengths for each communication to reduce the application execution time and ii) tune the laser driver current to provide just-enough optical power to meet the application BER requirements. This multi-objective optimization problem is solved using the Non-dominated Sorting Genetic Algorithm II (NSGA-II) [8], which results in a set of solutions on a Pareto front. From the set of Pareto points, the designer can select the most appropriate solution.

This paper is organized as follows. Section 2 presents the related work. Section 3 introduces the considered architecture model. The proposed methodology is detailed in Section 4 while Section 5 presents results. Finally, Section 6 concludes this paper.

\section{Related Work}

The high-bandwidth available in $\mathrm{ONoC}$ is directly related to Wavelengths Division Multiplexing (WDM), which allows propagating multiple signals simultaneously on a same waveguide. In WDM interconnect, Wavelength Allocation (WA)

plays an important role since it allows linking application level communications 
to resource allocation at the hardware level. In [9], Zang et al. distinguish dynamic allocation from static allocation: in the former case, the light-paths are set-up on demand at run-time while, in the latter case, the light-path requests are known in advance, which allows for wavelengths to be allocated using off-line methods. For this purpose, heuristic algorithms have been proposed [10] and include Random Wavelength Assignment, First-Fit, Most-Used and Least-Used algorithms. Most of these approaches aim to reduce the interconnect contention in order to reduce communication latency.

Different from their off-chip counterparts, on-chip interconnects suffer from very specific challenges related to WA. For instance, photonics devices such a microring-resonators (MR) lead to undesirable mode coupling between adjacent wavelengths in photodetectors, which generates crosstalk noise and impact ONoC performance [11]. Many studies have been carried out to estimate worstcase and average crosstalk noise in various ONoC topologies [12]. Furthermore, ONoC-specific WA methodology have been investigated in [13] [14]. These approaches rely on passive MR: no arbitration is needed to reserve optical paths before data transmission. However, these approaches suffer from a lack of scalability, are application specific and do not consider crosstalk noise in ONoC. Recently, a mapping tool has been proposed to improve the Signal to Noise Ratio (SNR) by reducing the number of communications sharing a waveguide [15]. Chittamuru et al. propose a crosstalk mitigation technique to increase channel spacing between adjacent wavelengths in Dense WDM (DWDM) [11]. Compared to these works, our methodology aims at configuring the laser output power at different levels according to the communication requirements. This strategy leads to a reduction of overall power needs and can be use together with the aforementioned approaches.

Lasers are responsible for a significant part of ONoC power consumption. Most architectures rely on the use of off-chip laser sources, which is a more mature technology compared to on-chip lasers. However, using off-chip laser requires to keep the lasers permanently ON once the system is booted. The unnecessary static power consumed under low traffic limits the energy/bit ONoC figures. For this purpose, Zhou et al. [16] proposed a prediction strategy based on network usage to modulate the laser power: the laser power is adjusted according to predictions based on previous activity. On-chip lasers have the potential to overcome static power in ONoC thanks to the reduction of coupling loss and to a simplified packaging. Vertical Cavity Surface Emitting Lasers (VCSELs) are cost-effective solutions and can be integrated due to their low threshold and driving current [17]. Furthermore, VCSELs based on double set of $\mathrm{Si} / \mathrm{SiO}_{2}$ photonics crystal mirror (PCM) are CMOS-compatible [18]. Commercial VCSELs can emit optical power of the order of a few $\mathrm{mW}$ around $850 \mathrm{~nm}$ with approximately $40 \%$ efficiency [19]. On-chip lasers can directly be turned ON or OFF, depending on the actual communication requirements [20] [21]. As detailed in [22], on-chip lasers operate in three modes: 1) OFF, 2) linear (the output optical power is proportional to the injected current) and 3) saturation. In [23], a feedback loop configures the laser power in the linear mode by using a 6-bit DAC. However, this solution suffers from a poor scalability due to the 


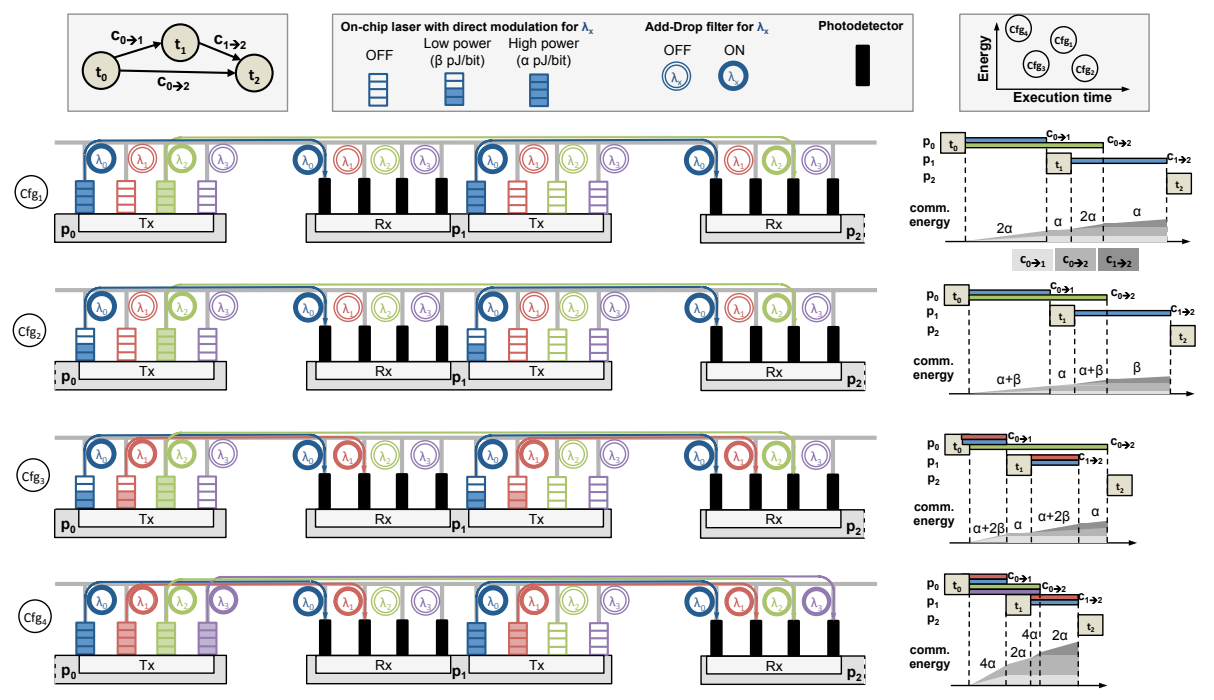

Figure 1: Chameleon optical interconnect allows for configuring the wavelengths allocated to communications and the laser output power.

need to estimate the BER on the receiver side and to the required feedback control. Furthermore, their is no analysis of the penalty induced by the BER estimation. In order to accurately reduce the power, Chen et al. proposed an on-off control strategy to maximize the energy efficiency [24]. The optical links are dedicated to access memory (L2 caches), which can be deactivated to save power. This method can be efficiently combined to the laser sharing and placing strategy they propose to improve the ONoC energy efficiency [25]. In [26], a CMOS driver is designed to configure the laser power to OFF, standby, midand full-power modes. They also consider a time and power penalty for OFFmode and standby-mode respectively. Our approach further extends this work by considering additional laser output power levels and by investigating their impact on the ONoC energy efficiency.

\section{Energy and Performance Aware Nanophotonic Interconnect}

This section introduces the architecture we consider and the reconfiguration features it provides. We then present the loss model considered in the paper. 


\subsection{Combined Wavelength Allocation and Laser Power Configuration}

The aim of this work is to identify, at design time, a set of ONoC configurations that will be embedded in a system and deployed at run time. Figure 1 illustrates the key concepts we investigate. Applications are represented as a Directed Acyclic Graph (DAG). In this example, we assume that tasks $t_{0}, t_{1}$ and $t_{2}$ are mapped onto processors $p_{0}, p_{1}$ and $p_{2}$, respectively. Communications $c_{0 \rightarrow 1}$, $c_{0 \rightarrow 2}$ and $c_{1 \rightarrow 2}$ are implemented using an $\mathrm{ONoC}$ which is configured according to execution performance and energy requirements.

The following illustrates four configurations:

- Baseline configuration $($ In this configuration, a single wavelength is allocated to each communication and the activated lasers are configured to emit high power optical signals. In the figure, lasers at wavelengths $\lambda_{0}$ (blue) and $\lambda_{2}$ (green) are allocated to $c_{0 \rightarrow 1}$ and $c_{0 \rightarrow 2}$ respectively (we assume a direct OOK modulation, i.e. no modulator is needed). The signals are injected into a waveguide using Microring Resonators (MRs) tuned to align their resonant wavelengths with signal wavelengths (ON state). The signals propagate along the waveguide until they reach their destination: signals at wavelength $\lambda_{0}$ and $\lambda_{2}$ are ejected in $p_{1}$ and $p_{2}$ interfaces respectively (the corresponding MRs in the interfaces are set to the ON state). Since $\lambda_{0}$ is used only from $p_{0}$ to $p_{1}$, it is reused to implement $c_{1 \rightarrow 2}$, thus maximizing the wavelength occupation in the waveguide. In this example, we assume that the targeted BER is reached for the all communications. The right hand-side of the figure illustrates the task and communication schedule: $p_{1}$ starts executing $t_{1}$ once all the data have been received from $p_{0}$ and then send processed data to $p_{2} . p_{2}$ first receives data from $p_{0}$ but the execution of $t_{2}$ is delayed until data from $p_{1}$ have been received. We also represent the $\mathrm{ONoC}$ communication energy under the task schedule: since the lasers are configured to output the maximum optical power, each communication consumes $\alpha$ pJ per bit transmitted.

- Low power configuration loads to the same communication scheme previously described. However, we assume that a lower optical power emitted by the lasers is enough to reach the targeted BER for $c_{0 \rightarrow 1}$ and $c_{1 \rightarrow 2}$ (1 hop each), while $c_{0 \rightarrow 2}$ still needs a high optical power due to the high losses experienced by the signal (2 hops). While the lower optical powers for $c_{0 \rightarrow 1}$ and $c_{1 \rightarrow 2}$ do not impact the task schedule, the energy consumption is reduced since it depends on both $\alpha$ and $\beta \mathrm{pJ} / \mathrm{bit}$ (obviously, $\alpha>\beta)$.

- Intermediate configuration implies a higher bandwidth allocated to $c_{0 \rightarrow 1}$ and $c_{1 \rightarrow 2}$. Indeed, both communications are implemented using $\lambda_{0}$ and $\lambda_{1}$ (red) which leads to $50 \%$ reduction in the communication latency. Despite the three simultaneous communications on the waveguide 
and hence the possibly higher crosstalk, we assume that the BER requirement is still met when $\lambda_{0}$ and $\lambda_{1}$ lasers are configured to their low-power mode. As depicted on the figure, $t_{1}$ and $t_{2}$ start executing earlier, which allows reducing the execution time compared to configurations ${ }^{\circ}$ and ${ }^{\circ}$. However, one can expect a higher increase of communication energy due to the laser thresholds current and voltage (i.e., $2 \times \beta>\alpha$ ).

- High performance configuration leads to a higher bandwidth for $c_{0 \rightarrow 2}$ since both $\lambda_{2}$ and $\lambda_{3}$ (purple) are allocated to the data transfer. Due to the high propagation distance ( 2 hops), $\lambda_{3}$ laser is also set to the high power mode. Furthermore, this configuration implies four simultaneous communications, which severely increases the crosstalk. This calls for a high optical power on all the communications, i.e. all the activated lasers are set to the high power mode, even those experiencing relatively low losses (1 hop). Hence, as depicted on the task schedule, the higher bandwidth for $c_{0 \rightarrow 2}$ allows for reducing the application execution time but significantly increases the communication energy.

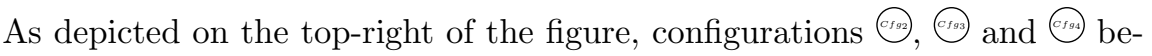
long to a Pareto front; the suitability of each configuration thus depends on the execution context (e.g. power and real time constraints). The network configurations are handled by a Configuration Manager that adapts ONoC configuration to satisfy application and execution context requirements. For instance, a constraint may be an execution deadline for real time systems, or energy minimization for battery powered systems. The run-time management of the $\mathrm{ONoC}$ configuration is not addressed in this paper. Moreover, task mapping and task relocation, which are complementary optimization techniques, are not investigated here.

\subsection{Interface Design}

Figure 2 illustrates an interface receiver for a 4-wavelength ONoC example. For case (a), we assume an interface receiving two signals on wavelengths $\lambda_{0}$ (blue) and $\lambda_{1}$ (red), i.e. blue and red MRs are set to the ON state. For this purpose, voltage tuning is applied to shift by $\Delta \lambda \mathrm{nm}$ the resonant wavelength of these MRs, that are eventually aligned with the signal wavelengths. Blue and red signals are thus dropped from the horizontal waveguide and reach photodetectors from where OE conversions are carried out. However, blue MR also drops a part of the red signal (dashed red arrow in the figure), which leads to crosstalk noise. The crosstalk power received by the photodetector depends on the MR drop spectrum, taking into account the resonances of the adjacent Free Spectral Ranges (FSR): the closer the red signals to the blue MR resonant wavelength, the higher the transmission. For case (a), the crosstalk is potentially high since the signals are transmitted using adjacent wavelengths [27]. To reduce the crosstalk, one can communicate using higher wavelength spacing between the signals. For case (b), we select wavelength $\lambda_{0}$ and $\lambda_{2}$ (green), which leads to a 

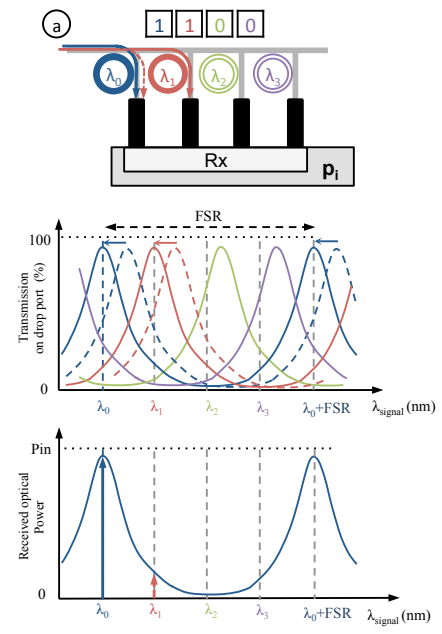
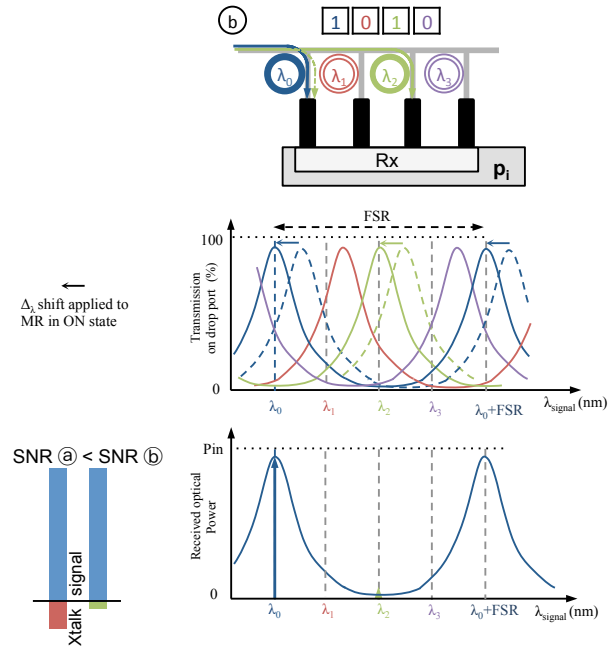

Figure 2: Receiver: the SNR depends on the wavelengths used to communicate.

lower crosstalk on the photodetector and thus to a higher SNR. Obviously, a high SNR helps improving the energy efficiency of the system since, for a given targeted BER, it allows for reducing the optical power emitted by lasers and hence their power consumption.

The model used for the MR considers no overhead in terms of time nor energy. Indeed, even if switching on or off can take time and require power, we assume that the $\mathrm{ONoC}$ will be used only for long communications. To keep the approach realistic when compared to solutions that do not involve interconnect reconfigurations, we target streaming applications, for which a significant amount of data are transmitted for each communication, i.e. the latency and dynamic power overhead used for the reconfiguration are negligible.

The optical signals are emitted using on-chip VCSELs with direct modulation. The optical power depends on the laser modulation current, as illustrated in Figure 3.a. In the OFF state, there is no current and hence no light emission. If the laser is likely to be used for data transmission, the current is set to $i_{\text {bias }}$, which corresponds to the threshold current from which light can be emitted. Since a direction modulation scheme is used, the data to be transmitted modulate the laser current as follows: data 0 leads to $i_{\text {bias }}$ (no light emission) and data 1 leads to a higher current (light emission). In this work, we assume a driver allowing to configure the laser current for the transmission of data 1 . The design complexity of the driver depends on the number of configurable laser power levels $\left(N P_{l v l}\right) . N P_{l v l}=4$ allows for selecting the modulation current for the transmission of data 1 as follow: $i_{1}, i_{2}, i_{3}$ and $i_{4}$ generate optical signals at $200 \mu W, 400 \mu W, 600 \mu W$ and $800 \mu W$, respectively. $N P_{l v l}=2$ leads to a reduced driver complexity but only allows for emitting optical signals at $400 \mu W$ and $800 \mu W . N P_{l v l}=1$ is the baseline scenario, with only $800 \mu W$ for 

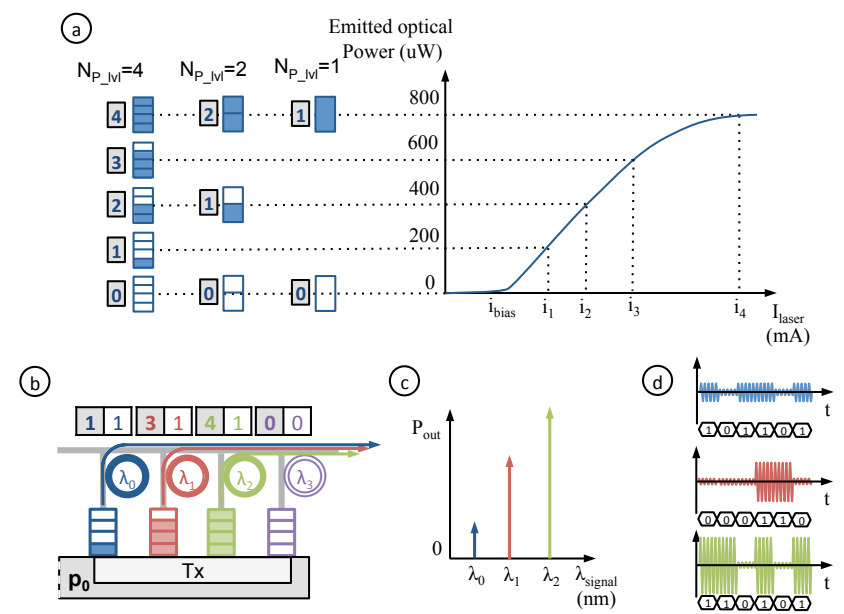

Figure 3: Configurable laser output power in the transmitter.

emitting data 1. Obviously, the higher $N P_{l v l}$, the more the ONoC is adaptable to application requirements (BER) and communication context (crosstalk and propagation losses). However, this adaptability comes with a higher design complexity, from which the study is part of our future work.

Figure 3.b represents an interface transceiver for a 4 -wavelength network and for $N P_{l v l}=4$. In this example, blue, red and green lasers emit a $200 \mu W$ (configuration 1), $600 \mu W$ (3) and $800 \mu W$ (4); the corresponding MRs are set to the ON state to inject the signals into the horizontal waveguide (MR configuration is 1). Purple laser and its MR are OFF (configuration 0). Figure 3.c illustrates the distribution of the signal wavelengths in the spectrum and their power level. Figure 3.d represents the OOK modulation for data stream examples. The maximum output power is a designer choice regarding the targeted laser technology and the architecture requirements. In our simulations, we define it by considering the worst case regarding losses and communication configurations.

\subsection{BER Estimation}

The following presents the loss model we use to evaluate the BER. It relies on the MR model proposed [28], which allows estimating transmission $\Phi_{d B}^{d}$ and $\Phi_{d B}^{t}$ (i.e. the transmission on drop port and through port respectively) according to the distance between a signal at $\lambda_{i}$ and a MR at resonant wavelength $\lambda_{m}$. For one resonance centered on $\lambda_{m}$, this model is as follow:

$$
\begin{gathered}
\Phi_{d B}^{d}\left(\lambda_{i}, \lambda_{m}\right)=\frac{P_{D}[i]}{P_{\text {input }}[i]}=\frac{\delta^{2}}{\left(\lambda_{i}-\lambda_{m}\right)^{2}} \\
\Phi_{d B}^{t}\left(\lambda_{i}, \lambda_{m}\right)=1-\Phi_{d B}^{d}\left(\lambda_{i}, \lambda_{m}\right)
\end{gathered}
$$


where $2 \delta$ is the $-3 d B$ bandwidth of the MR, which can be defined as $Q=\frac{\lambda_{m}}{2 \delta}$, with $\mathrm{Q}$ the quality factor of the MR. $P_{\text {input }}[i]$ is the input optical power of signal at $\lambda_{i}$ and $P_{D}[i]$ is the optical power on the drop port. As previously stated, the signal transmission of $\mathrm{MR}_{\lambda_{m}}$ depends on its state: OFF-state and ON-state lead to $\lambda_{m}+\Delta \lambda$ and $\lambda_{m}$ resonant wavelengths respectively.

Regarding the losses of a wavelength crossing a MR, we extended the aforementioned model by considering three resonances: the main at $\lambda_{m}$, and two mores at $\lambda_{m}+F S R$ and $\lambda_{m}-F S R$. FSR (Free Spectral Range) corresponds to the difference between two successive MR's resonant wavelengths.

Hence, on the through port, the signal power $P_{T, M o n_{\lambda_{m}}}[i]$ at $\lambda_{i}$ for a MR in the ON-state is defined as

$$
\begin{aligned}
P_{T, \text { Mon }_{\lambda_{m}}}[i]= & P_{\text {input }}[i]+\Phi_{d B}^{t}\left(\lambda_{i}, \lambda_{m}-F S R\right)+\Phi_{d B}^{t}\left(\lambda_{i}, \lambda_{m}\right)+\Phi_{d B}^{t}\left(\lambda_{i}, \lambda_{m}+F S R ß ß\right) \\
& \forall i \in\left\{1, \ldots, N_{\lambda}\right\}
\end{aligned}
$$

and the signal power $P_{T, M o f f_{\lambda_{m}}}[i]$ at $\lambda_{i}$ for a MR in the OFF-state is defined as

$$
\begin{aligned}
P_{T, M o f f_{\lambda_{m}}}[i]= & P_{\text {input }}[i]+\Phi_{d B}^{t}\left(\lambda_{i}, \lambda_{m}+\Delta \lambda-F S R\right)+\Phi_{d B}^{t}\left(\lambda_{i}, \lambda_{m}+\Delta \lambda\right)+\Phi_{d B}^{t}\left(\lambda_{i}, \lambda_{m}+\Delta \lambda+F S(\text { (स) })\right. \\
& \forall i \in\left\{1, \ldots, N_{\lambda}\right\}
\end{aligned}
$$

The transmissions on the drop ports, i.e. $P_{D, M o n_{\lambda_{m}}}[i]$ and $P_{D, M o f f_{\lambda_{m}}}[i]$ are obtained similarly.

In order to estimate the SNR on a photodetector, we estimate the losses experienced by the all the transmitting signals, from the laser sources to the destinations, taking into account the states of the crossed MRs. This leads to Eq. 5 and Eq. 6, which respectively correspond to the received signal power $\left(P_{\lambda_{m}}^{s}\right)$ and crosstalk power $\left(P_{\lambda_{m}}^{n}\right)$ on a photodetector connected to a MR at wavelength $\lambda_{m}$.

$$
\begin{gathered}
P_{\lambda_{m}}^{s}=P_{\text {laser }}+L_{M R, o n}[m]+L_{M R, o f f}[m]+L_{P}[m] \\
P_{\lambda_{m}}^{n}=\sum_{j \neq m}^{j \in\{M\}} P_{\text {laser }}+L_{M R, o n}[j]+L_{M R, o f f}[j]+L_{P}[j]
\end{gathered}
$$

where $\{M\}$ is the set of signals at wavelength $\lambda_{j} \neq \lambda_{m} . P_{l a s e r}$ is the laser output power. $L_{M R, o n}[j]$ and $L_{M R, o f f}[j]$ are the losses experienced by signals due OFF-state and ON-state MRs crossing. $L_{P}[j]$ is the propagation loss.

SNR and and BER are defined by Eq. 7 and Eq. 8 respctively.

$$
\begin{gathered}
S N R_{\lambda_{m}}=\frac{P_{\lambda_{m}}^{s}}{P_{\lambda_{m}}^{n}+P_{p d}^{n}} \\
B E R=\frac{1}{2} \operatorname{erfc}\left(\frac{S N R}{2 \sqrt{2}}\right)
\end{gathered}
$$
tion.

Where $P_{p d}^{n}$ is photodetector noise and erfc is the complementary error func- 


\section{Proposed Methodology}

In this section, we present the proposed design flow, which aims at optimizing the $\mathrm{ONoC}$ configuration to execute a given application.

\subsection{Overview of the Design Flow}

Figure 4 illustrates the design flow generating ONoC configurations according to user specifications. The flow takes as inputs an application mapped onto a $3 \mathrm{D}$ architecture. The application is modeled as a DAG characterized by task execution times, amount of data transmitted between tasks, and minimum BER to be reached. The architecture includes an ONoC implemented on top of processing cores and characterized by a topology, a number of wavelengths and waveguides. The $\mathrm{ONoC}$ allows for cores to communicate with each other using optical signals, which is achieved using $\mathrm{E} / \mathrm{O}$ and $\mathrm{O} / \mathrm{E}$ conversion. The interfaces are crossed by waveguides propagating the optical signals using WDM in both clockwise (C) and counter-clockwise (CC) directions, which reduces the maximum communication distance. The mapping of the tasks on the cores gives the communications in the ONoC. The design flow relies on technological parameters since they impact the performance of optical communications. Instance of parameters are photodetectors sensitivity, waveguide losses and MR transmission. Regarding the laser, we take the data-rate, the efficiency, the maximum output power, and the number of power levels available into account.

The aim of the flow is to optimize both power consumption and application execution time. For this purpose, we explore the number of wavelengths allocated to each communication, i.e. the bandwidth and the power level for each laser source. A multi-objective optimization method is implemented using a genetic algorithm, which allows extracting solutions on a Pareto front. Comparison with other heuristics that could be used to solve this optimization problem is out of the scope of this paper. In our genetic algorithm, the ONoC configurations (i.e. the individuals) are represented by chromosomes and the genes encode both wavelength allocations and laser power levels. Classical crossover and mutation operators are applied to evolve the initial population. Constraints validate the configurations by ensuring that i) BER requirement is reached and ii) no wavelength is used more than once on a same waveguide segment. For each valid individual, a simulation is carried out to estimate application execution time and total $\mathrm{ONoC}$ energy consumption (i.e. the algorithm fitness functions). The resulting $\mathrm{ONoC}$ configurations are thus made available to the designer for final selection. Furthermore, since all the configurations are non-dominated on at least one metric, multiple configurations could be embedded in the system in order to be loaded at run-time according to the execution

context (e.g. high performance and low power). This is out of the scope of the paper and is part of our future work. 


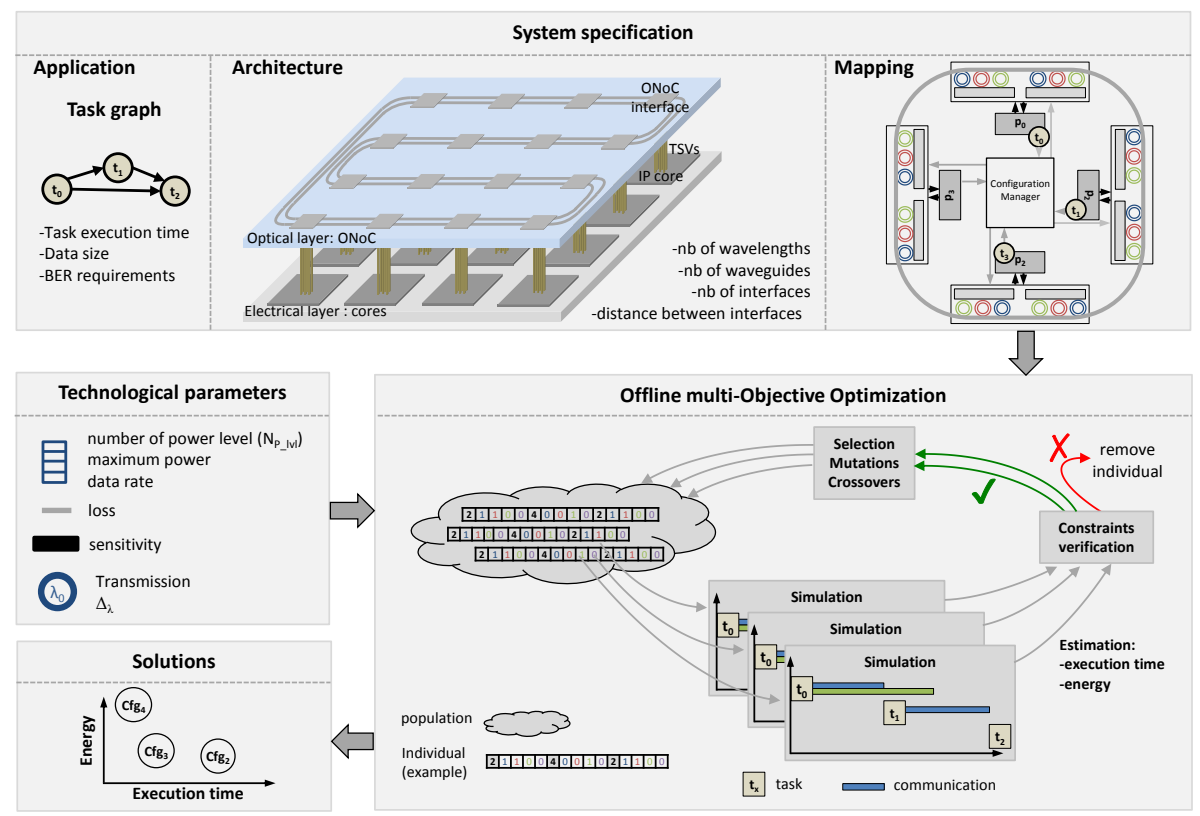

Figure 4: Multi-objective design space exploration.

\subsection{Individual Coding}

Figure 5 illustrates individual coding and the corresponding ONoC configuration. Since we reuse configuration example from Figure 1, we assume three interfaces, four wavelengths (i.e. four lasers per interface) and four laser output power levels. Tasks $t_{0}, t_{1}$ and $t_{2}$ are mapped on processor $p_{0}, p_{1}$, and $p_{2}$, respectively, which leads to optical communications between $p_{0}$ and $p_{1}\left(c_{0 \rightarrow 1}\right)$, $p_{0}$ and $p_{2}\left(c_{0 \rightarrow 2}\right), p_{1}$ and $p_{2}\left(c_{1 \rightarrow 2}\right)$. The chromosome is divided into as many parts as there are communications (three in the example). The first gene of each chromosome part gives the selected laser output power level; it is an integer value corresponding to the laser configuration (see Figure 3 for details). The following genes correspond to the wavelengths utilization: value 0 or 1 indicates that a wavelength is used or unused, respectively. In the example, both $c_{0 \rightarrow 1}$ and $c_{1 \rightarrow 2}$ use $\lambda_{0}$ and $\lambda_{1}$ while $c_{0 \rightarrow 2}$ is implemented using only $\lambda_{2}$. Obviously, the more wavelengths are allocated for a given communication, the higher the bandwidth.

The configuration of each interface is obtained as follows. First, optical channels are open by switching to the ON state the MRs involved in communications (i.e. MRs localized in the transmitter Tx of the source processor and the receiver $\mathrm{Rx}$ of the destination). Then, the power of the optical signals propagating through the channels is defined according to the selected lasers power level. In the example, at the transmitter of $p_{0}$ interface, three MRs are turned ON to implement communications $c_{0 \rightarrow 1}\left(\lambda_{0}\right.$ and $\left.\lambda_{1}\right)$ and $c_{0 \rightarrow 2}\left(\lambda_{2}\right)$. On the 


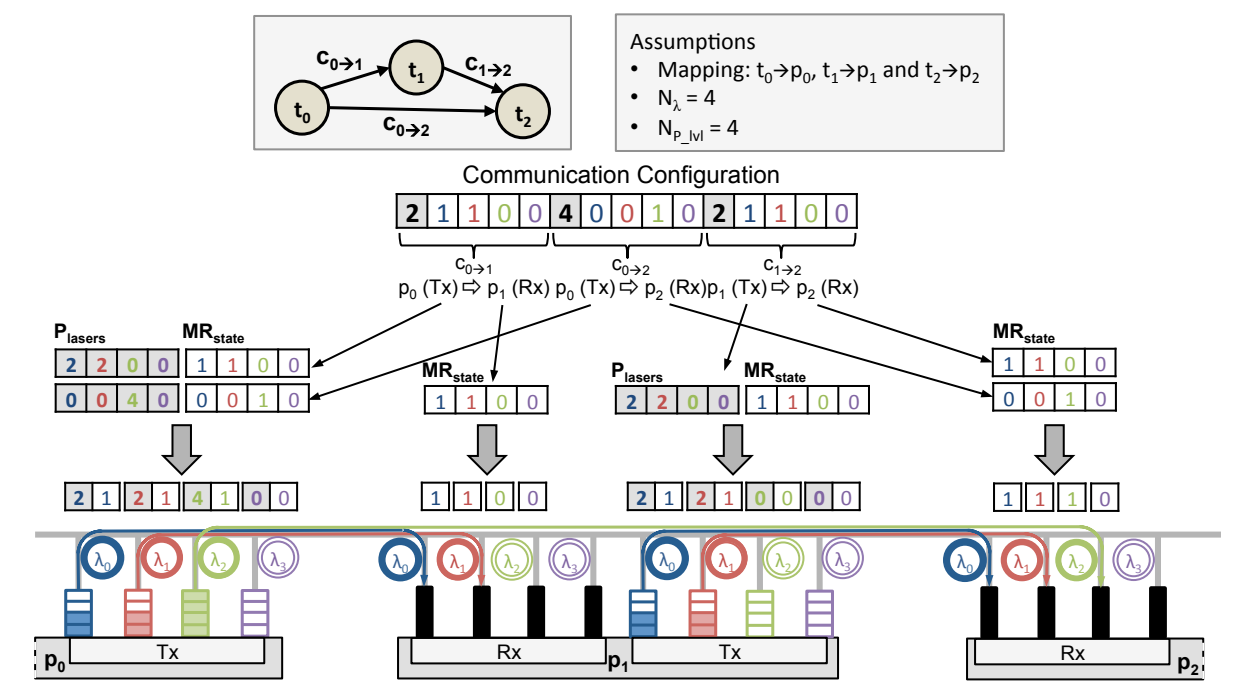

Figure 5: Individual coding structure example and the corresponding ONoC configuration

receiver side, the MRs corresponding to $\lambda_{0}$ and $\lambda_{1}$ are set to the ON state in $p_{1}$ interface, while the MR corresponding to $\lambda_{2}$ remains OFF to let the signal reaching $p_{2}$ interface were it will be dropped. In the chromosome part dedicated to $c_{0 \rightarrow 1}$, the laser output power level is set to 2 : in $p_{0}$ interface, the lasers emitting at wavelength $\lambda_{0}$ and $\lambda_{1}$ are set to $50 \%$ of the maximum power. In the same interface, laser at $\lambda_{2}$ is set to $100 \%$ to match value 4 in corresponding gene for $c_{0 \rightarrow 2}$.

The size of an interface configuration is rather small since it requires only $N_{\lambda} \times\left(\log _{2}\left(N_{\text {laser }}\right)+2\right)$ bits. Hence, one can easily and rapidly load predefined configurations from a dedicated memory in order to execute new applications or to adapt to an evolution of the execution context. Changing the ONoC configuration without loss of data can be simply achieved by waiting for the end of ongoing transmissions and by possibly setting the optical devices to the OFF state. However, lower latency reconfiguration and tighter adaptation to the evolution could be carried out, for instance, to maintain existing optical channels. This could be carried out at run-time by an operating system, which is currently under investigation but remains out of the scope of the paper.

This example illustrates the flexibility of our approach, which combines bandwidth allocation and laser output power tuning features. Indeed, one can allocate high bandwidth channels to time critical communications or minimize bandwidth for lower constrained communications. Tuning the laser output power ideally complements bandwidth allocation flexibility by i) alleviating crosstalk effect and by ii) allowing energy proportional optical communications. 


\subsection{Performance Evaluation}

During the optimization process, the individuals are evaluated using fast simulations executed with Python. The simulator provides estimations of i) the application execution time, considering the $\mathrm{ONoC}$ allocated bandwidth, and ii) the $\mathrm{ONoC}$ power consumption, considering the selected output power of the laser sources. A centralized controller configures the optical devices as the application is executing. It is responsible for opening optical channels when communications are initiated and for closing them when data transfers terminate. This is illustrated in Figure 6, which represents the evolution of the optical devices states during the execution of a 4-task application on a 4-processor architecture. In this example, we assume three wavelengths per waveguide and we consider two bidirectional waveguides, i.e. waveguides propagate optical signals in opposite directions.

Figure 6.a represents the input task graph and figure 6.b is a communication graph obtained considering i) the mapping on the processors and ii) the bandwidth allocated to each communication. While a complete communication task would consider all possible communication end-time sequence scenarios (for instance, $c_{1 \rightarrow 2}$ can terminate before or after $c_{1 \rightarrow 3}$ ), we consider a reduced graph for which communication times are estimated. It is worth mentioning that a reduced graph improves the scalability of our approach by simplifying the design of the controller and by reducing the memory footprint. Following the method detailed in Section 4.2, each state of the communication graph is associated to a configuration $Q$. Figure 6.c summarizes the possible configurations of the transceivers $\left(T x_{i}\right)$ and the receivers $\left(R x_{i}\right)$ placed along clockwise and counterclockwise waveguides (only values different from 0 are represented for clarity purpose). The configurations are stored in a dedicated memory in the controller and are used at run-time.

During the simulation, each time the $\mathrm{ONoC}$ is reconfigured to start a new communication, the simulator achieves the following operations:

- The communication end-time is computed and an event is added to the simulator event list. The communication time depends on the number of allocated wavelengths (parameter explored during the optimization), the number of data to be transmitted (given in the task graph) and the considered laser data rate (technological parameter).

- The energy required by the starting communication is added to the total ONoC energy consumption. In addition to previously listed parameters, the energy consumption also depends on the laser output power (explored parameter) and on its power model (technological parameter).

- The BER is evaluated for all ongoing communications (i.e. not only the newly added since higher crosstalk can occur and may impact ongoing communications) using the analytical model proposed in [29]. In case the BER requirement is not met, the simulation stops and the individual is removed. This also occurs if the same wavelength is used by multiple communications simultaneously on a waveguide segment. 
Figure 6.d illustrates a resulting execution trace for the considered example. $p_{0}$ sends a control signal to the configuration manager when $t_{0}$ execution ends and when data involved in $c_{0 \rightarrow 1}$ are ready for transfer. The manager switches from configuration $Q_{\text {init }}$ (all the optical devices are in the OFF-state, i.e. there is no communication in the $\mathrm{ONoC}$ ) to $Q_{A}: T x_{0}$ and $R x_{1}$ are reconfigured with the values stored in the configuration memory. In the example, three clockwise wavelengths are allocated, as defined in the first line of table in Figure 6.c. For low-latency purpose, the $\mathrm{ONoC}$ is reconfigured in parallel, which is implemented using dedicated electrical wires connecting the controller to the optical devices. Once the new configuration is loaded, the data transfer starts and, once it ends, another control signal is sent to the configuration manager to disable the communication channels. For this purpose, a new configuration $\left(Q_{\text {init }}\right.$ in the example) is loaded i.e. the lasers and the MR involved in the communication are turned OFF. Figure 6.e illustrates the ONoC configuration to carry out communications $c_{1 \rightarrow 3}$ and $c_{1 \rightarrow 2}$ clockwise and counter-clockwise respectively (the laser output powers are not illustrated for the sake of clarity). In the example, the directions are defined by the smallest distance between the source and destination processors. Since $c_{1 \rightarrow 2}$ communication terminates first, the corresponding optical devices are turned OFF. This is achieved without affecting the configuration of the devices used for still ongoing $c_{1 \rightarrow 3}$ (reconfiguration $\left.Q_{B 2} \rightarrow Q_{B 3}\right)$. Similarly, the reconfiguration of the interfaces to implement a new communication has no impact on the ongoing communications: in the example illustrated in Figure 6.f, red signal on $p_{3}$ to $p_{2}$ waveguide segment is used for $c_{2 \rightarrow 3}$ while $c_{1 \rightarrow 3}$ is carried out using green and blue wavelengths (reconfiguration $Q_{B 3} \rightarrow Q_{B 4}$ ).

\section{Results}

This section presents evaluations of the proposed method. We first discuss on a case study to illustrate the impact on performance provided by our approach. We then analyze the scalability of our approach on more complex applications.

\subsection{ONoC Design Parameters}

In this study, we investigate the impact of two key ONoC design parameters, namely the number of laser power levels and the number of the wavelengths, on performance and power consumption. For this purpose, we assume first the simple task graph illustrated in Figure 7. The task computation times and the volume of transmitted data are given in kilo clock cycles (kcc) and kilobit (kb) respectively. Regarding the architecture, we assume a $4 \times 4$ ONoC with $1 \mathrm{Ghz}$ processor frequency and $10 \mathrm{~Gb} / \mathrm{sec}$ laser data rate. The maximum electrical power of the laser is set to $4 \mathrm{~mW}$ and the remaining architectural parameters are given in Table 1. The mapping we assume is illustrated on the right hand side of the figure. The multi-objective optimization algorithm is set to iterate 800 times with a population size of 500 individuals. Regarding the BER estimation, 

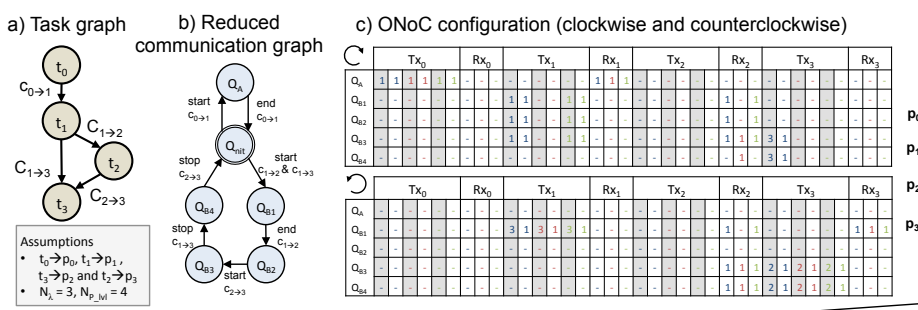

d) Simulation results
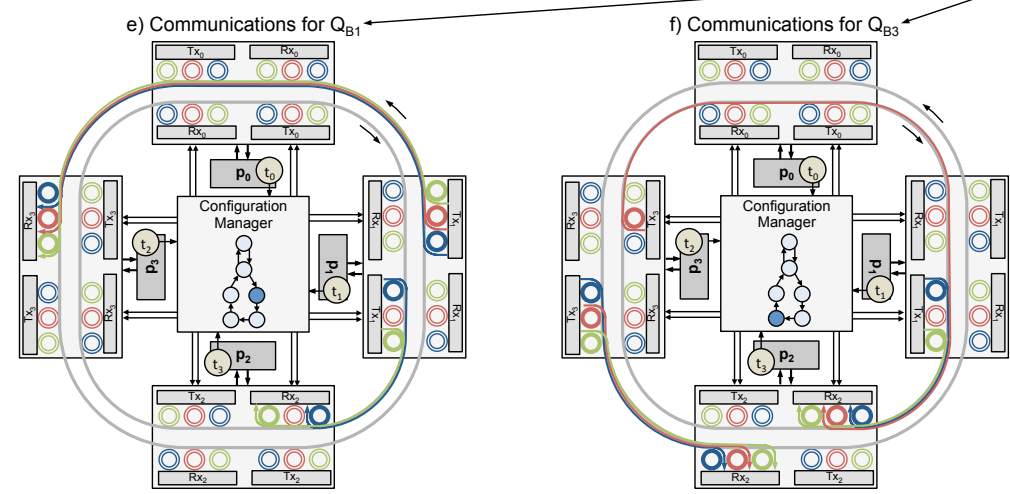

Figure 6: Performance estimation using an event-based simulator and run-time reconfiguration of the $\mathrm{ONoC}$.

we assume the model detailed in Section 3.3 and the technological parameters defined in Table 1.

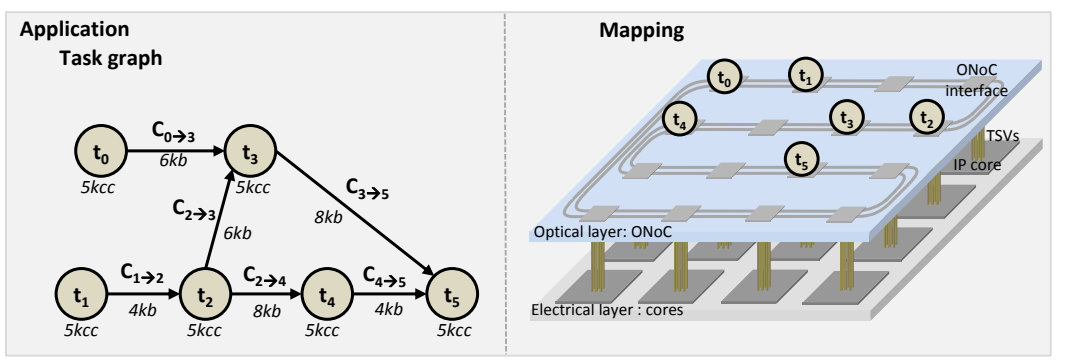

Figure 7: Characterized task graph and its mapping on a $4 \times 4$ ONoC.

\subsubsection{Number of laser power levels}

In this study, we investigate the impact of the number of laser power levels on the $\mathrm{ONoC}$ energy consumption. For this purpose, we assume a single waveguide with $N_{\lambda}=8$ and we assume three values for $N P_{l v l}: 3,5$ and 7 . As we consider electrical power lasers from $1 \mathrm{~mW}$ to $4 \mathrm{~mW}$, the configurable powers, in $\mathrm{mW}$, 
Table 1: Technological parameters.

\begin{tabular}{|c|c|c|}
\hline Parameter & Value & Ref \\
\hline Waveguide propagation loss & $-0.274 \mathrm{~dB} / \mathrm{cm}$ & {$[30]$} \\
\hline Photodetector sensitivity & $-20 \mathrm{dBm}$ & {$[31]$} \\
\hline Laser efficiency & $15 \%$ & {$[31]$} \\
\hline$\Delta \lambda$ & $0.4 \mathrm{~nm}$ & {$[27]$} \\
\hline FSR & $8 \mathrm{~nm}$ & {$[27]$} \\
\hline$-3 d B$ MR bandwidth & $0.26 \mathrm{~nm}$ & {$[27]$} \\
\hline
\end{tabular}

are: $[1,2.5,4]$ for $N P_{l v l}=3,[1,1.75,2.5,3.25,4]$ for $N P_{l v l}=5$, and $[1,1.5,2$, $2.5,3,3.5,4]$ for $N P_{l v l}=7$.

Figures 10(a) and 10(b) show the resulting solutions for targeted BER of $10^{-9}$ and $10^{-12}$, respectively. Each solution is characterized by the total laser energy consumption and the application execution time. The total laser energy is the sum of all the energy used by the lasers. In Figure 10-a, $N P_{l v l}=3$ leads to solutions ranging from $56 \mathrm{nj}$ to $63 \mathrm{nj}$ for the laser energy and from $26 \mathrm{kcc}$ to $24.2 \mathrm{kcc}$ for the execution time. These results highlight the Pareto front induced by the conflicting optimization of performance and low-power objectives. This trend is further increased for $N P_{l v l}=5$ since the execution time interval ranges from $30 \mathrm{kcc}$ to $24 \mathrm{kcc}$. It is important to notice that the energy consumption decreases since the optical signal power emitted by the laser can be more accurately adapted to compensate for the losses experienced by the optical signals and to reach the BER requirement. For instance, for the solutions characterized by $26 \mathrm{kcc}$ execution time, $N P_{l v l}=3$ and $N P_{l v l}=5$ lead to $56 \mathrm{nj}$ and $46 \mathrm{nj}$ energy consumption, respectively. The energy is further reduced to $45 \mathrm{nj}$ for $N P_{l v l}=7$, which allows for finer-grain configuration of the laser. Obviously, the higher $N P_{l v l}$, the closer to the optimal solution the results. However, this comes with a more complex design of the laser driver, which is out of the scope of the paper. These results demonstrate the need for configurable laser output power to improve both performance and energy consumption in ONoC based architectures. Figure 10(b) shows the same trends for a $10^{-12}$ BER. This set of configuration solutions can be used if more robust executions of the application are needed, but this comes with a higher energy consumption since the laser output power needs to be increased to improve the SNR. For instance, for the solutions characterized by $26 \mathrm{kcc}$ execution time and with $N P_{l v l}=7$, the required energy increases from $45 \mathrm{~nJ}$ to $51 \mathrm{nj}$ when a BER of $10^{-12}$ is targeted. This trend illustrates the opportunity introduced by our approach to consider Quality of Service in ONoC architectures.

\subsubsection{Number of wavelengths}

Figures 13(a) and 13(b) give the optimization results for $N_{\lambda}=4,6$, and 8 and with $N P_{l v l}=7$ and $N P_{l v l}=5$, respectively. The targeted BER is $10^{-12}$. The results first highlight that the higher the number of wavelengths, the higher the 


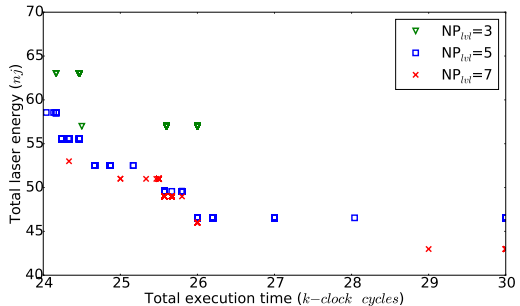

(a)

Figure 8: *

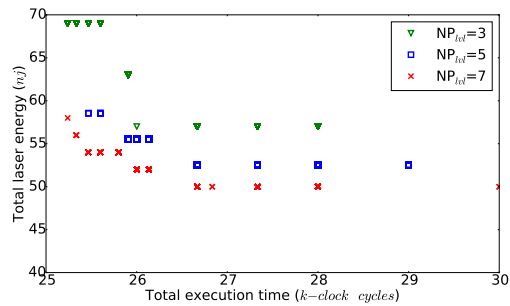

Figure 9: *

(b)

Figure 10: Optimization results: solutions are characterized by an execution time and an energy for $N P_{l v l}=3,5$, and 7 , for a targeted BER of (a) $10^{-9}$ and (b) $10^{-12}$.

number of solutions and the higher the diversity. For instance, for $N_{\lambda}=4$, the execution time of resulting solutions ranges from $30 \mathrm{kcc}$ to $28.3 \mathrm{kcc}$, while for $N_{\lambda}=8$ solutions ranges from $30 \mathrm{kcc}$ to $25.3 \mathrm{kcc}$. However, more wavelengths in the $\mathrm{ONoC}$ leads to a reduction of the energy efficiency, even for solutions in which few wavelengths are actually allocated (see solutions with a long execution time on the right part of the figures). Indeed, more wavelengths in the ONoC means more MRs in the ONIs, which increases the losses. The energy efficiency reduction is further accentuated when multiple wavelengths are assigned to implement the communications: solutions showing low execution time lead to inter-channel crosstalk effects that require higher laser output power. For instance in Figure 13(a), the configuration providing the lowest energy consumption with $N_{\lambda}=8$ requires $50 \mathrm{nj}$ and $30 \mathrm{kcc}$, while the lowest energy consumption with $N_{\lambda}=6$ requires only $43 \mathrm{nj}$ for the same execution time. The same execution time is synonym of the same number of allocated wavelength for each communication, meaning the same amount of dynamic power is used. Hence, the difference in terms of energy comes from the static losses due to the increase of MR numbers.

To summarize, designing an $\mathrm{ONoC}$ with more wavelengths increases the bandwidth allocated for each communication. However, this also reduces the energy efficiency due to higher propagation losses (more resonators are crossed) and higher crosstalk (the wavelength spacing is reduced).

\subsection{Wavelength Allocation and Laser Power Distribution}

In this section, we investigate the scalability of the approach using $\mathrm{ONoC}$ architectures with 16, 32 and 64 ONIs, that interconnect 64, 128, and 256 cores respectively. Each ONI is connected to a cluster of four electrically connected cores. We assume two waveguides, eight wavelengths per waveguide, and $N P_{l v l}=5$. For the $\mathrm{ONoC}$ with 64 cores the possible electrical laser power 


\begin{tabular}{|c|c|c|c|c|c|c|c|c|c|}
\hline & \multirow{3}{*}{$\begin{array}{l}\text { Graph } \\
\text { ID }\end{array}$} & \multirow{3}{*}{$\begin{array}{c}\text { Number } \\
\text { of } \\
\text { Tasks } \\
\end{array}$} & \multirow{3}{*}{$\begin{array}{c}\text { Number } \\
\text { of } \\
\text { Comm. }\end{array}$} & \multicolumn{6}{|c|}{ Energy (nj) } \\
\hline & & & & & Low Pow & & & High Per & \\
\hline & & & & ON-OFF & Proposed & Reduc. (\%) & ON-OFF & Proposed & Reduc. (\%) \\
\hline \multirow{9}{*}{$\begin{array}{l}64 \text { cores } \\
16 \text { ONI }\end{array}$} & TG 1 & 55 & 80 & 463 & 141 & 69.5 & 463 & 187 & 59.6 \\
\hline & TG 2 & 52 & 78 & 433 & 118 & 72.7 & 433 & 194 & 55.2 \\
\hline & TG 3 & 57 & 82 & 469 & 102 & 78.3 & 469 & 120 & 74.4 \\
\hline & TG 4 & 60 & 92 & 525 & 141 & 73.1 & 525 & 195 & 62.9 \\
\hline & TG 5 & 63 & 93 & 497 & 128 & 74.2 & 497 & 193 & 61.2 \\
\hline & TG 6 & 62 & 92 & 530 & 147 & 72.3 & 530 & 236 & 55.4 \\
\hline & TG 7 & 56 & 87 & 480 & 103 & 78.5 & 480 & 148 & 69.2 \\
\hline & TG 8 & 63 & 91 & 492 & 112 & 77.2 & 492 & 156 & 68.3 \\
\hline & Average & & & 486 & 124 & 74.5 & 486 & 179 & 63.3 \\
\hline \multirow{2}{*}{$\begin{array}{r}128 \text { cores } \\
32 \text { ONI } \\
\end{array}$} & TG 9 & 107 & 158 & 1346 & 253 & 81.2 & 1346 & 389 & 71.1 \\
\hline & TG 10 & 94 & 139 & 1145 & 213 & 81.4 & 1145 & 314 & 72.6 \\
\hline \multirow{2}{*}{$\begin{array}{r}256 \text { cores } \\
64 \text { ONI } \\
\end{array}$} & TG 9 & 107 & 158 & 2871.4 & 748.35 & 73.9 & 2871.4 & 1051 & 63.4 \\
\hline & TG 10 & 94 & 139 & 3055.2 & 654.4 & 78.6 & 3055.2 & 1153.4 & 62.2 \\
\hline
\end{tabular}

Table 2: Energy comparisons between $\mathrm{ON}-\mathrm{OFF}$ strategy $\left(N P_{l v l}=1\right)$ and our Proposed methodology with $N P_{l v l}=5$ for different task graphs.

\begin{tabular}{|c|c|c|c|c|c|c|c|c|c|}
\hline & \multirow{2}{*}{$\underset{\text { ID }}{\text { Graph }}$} & \multirow{2}{*}{$\begin{array}{c}\text { Number } \\
\text { of } \\
\text { Tasks }\end{array}$} & \multirow{2}{*}{$\begin{array}{c}\begin{array}{c}\text { Number } \\
\text { of }\end{array} \\
\text { Comm. }\end{array}$} & \multicolumn{3}{|c|}{ Energy (nj) } & \multicolumn{3}{|c|}{ Execution Time (kcc) } \\
\hline & & & & Low Power & High Perf. & Variation & Low Power & High Perf. & Variation \\
\hline \multirow{9}{*}{$\begin{array}{l}64 \text { cores } \\
16 \text { ONI }\end{array}$} & TG 1 & 55 & 80 & 141 & 187 & 1.33 & 41.2 & 23.8 & 1.73 \\
\hline & TG 2 & 52 & 78 & 118 & 194 & 1.64 & 37.9 & 23.4 & 1.62 \\
\hline & TG 3 & 57 & 82 & 102 & 120 & 1.18 & 39.9 & 21.7 & 1.84 \\
\hline & TG 4 & 60 & 92 & 141 & 195 & 1.38 & 37.3 & 23 & 1.62 \\
\hline & TG 5 & 63 & 93 & 128 & 193 & 1.51 & 41.2 & 21.8 & 1.89 \\
\hline & TG 6 & 62 & 92 & 147 & 236 & 1.61 & 43.2 & 24.7 & 1.75 \\
\hline & TG 7 & 56 & 87 & 103 & 148 & 1.44 & 31.7 & 20 & 1.57 \\
\hline & TG 8 & 63 & 91 & 112 & 156 & 1.39 & 37.5 & 23.4 & 1.60 \\
\hline & Average & & & 124 & 179 & 1.44 & 38.7 & 22.7 & 1.71 \\
\hline \multirow{2}{*}{$\begin{array}{r}128 \text { cores } \\
32 \text { ONI }\end{array}$} & TG 9 & 107 & 158 & 253 & 389 & 1.54 & 62.19 & 37.5 & 1.66 \\
\hline & TG 10 & 94 & 139 & 213 & 314 & 1.47 & 64.38 & 33.6 & 1.92 \\
\hline \multirow{2}{*}{$\begin{array}{c}256 \text { cores } \\
64 \text { ONI }\end{array}$} & TG 9 & 107 & 158 & 748.35 & 1051 & 1.40 & 64.38 & 38.1 & 1.69 \\
\hline & TG 10 & 94 & 139 & 654.4 & 1153.4 & 1.76 & 61.01 & 34.58 & 1.76 \\
\hline
\end{tabular}

Table 3: Energy and execution time for Low Power and High Performance wavelength allocations strategies. 


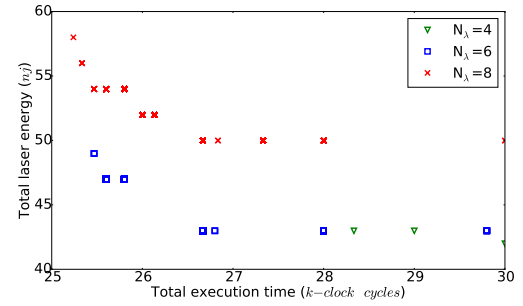

Figure 11: *

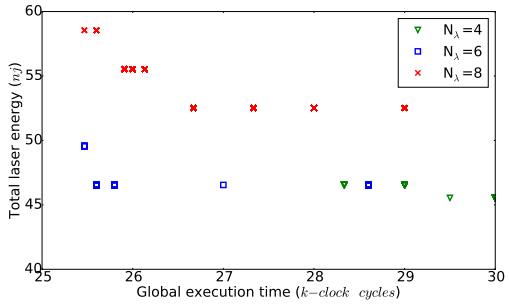

Figure 12: *

Figure 13: Optimization results for various number of wavelengths $N_{\lambda}$, and with (a) $N P_{l v l}=7$ and (b) $N P_{l v l}=5$.

values are $[2,4,6,8,10] \mathrm{mW}$, and for 128 cores the possible electrical laser power values are $[3,6,9,12,15] \mathrm{mW}$, while for 256 cores the laser power values are $[4,13,22,31,40] \mathrm{mW}$. Regarding the applications, we use a random task graph generator that provides applications including from 52 to 107 tasks and from 80 to 158 communications. The task execution time values are randomly selected between $[100,1000]$ cc and the communication volumes are randomly selected within $[100,1000]$ bytes range. The targeted BER is $10^{-9}$ and each task is randomly mapped on a dedicated core. As we assume shared memory within a same cluster, no latency is assumed for intra-cluster communications.

Tables 2 and 3 summarize the characteristics of the task graphs and the optimization results. Since our approach leads to a Pareto front, we only show the solutions with i) the lowest energy consumption (denoted Low Power in the table) and i) the lowest execution time (High Perf.). We also provide results for the baseline ON-OFF strategy (i.e. $N P_{l v l}=1$ ). Table 2 compares the energy consumption between ON-OFF strategy and our proposed method. Regarding the low-power solutions, our methods leads to $74.5 \%$ reduction on average in the energy consumption compared to the ON-OFF solutions. This is due to the laser output power which is adapted to satisfy the BER requirements. Indeed, for our proposed strategy, low Laser power levels are often selected to support communications, while in the ON-OFF strategy, only high Laser power level can be used. It is worth mentioning that the high-performance solutions resulting from our method also shows a significant $63 \%$ reduction on average in energy compared to the baseline strategy. We can note that for both strategies, because the number of wavelengths allocated for each task graph is the same, the execution time is unchanged.

As shown in Table 3, the solutions offer, on average, $44 \%$ energy variation and $71 \%$ execution time variation trade-offs for 64 cores architectures. As the results show, the execution time gain increases for larger architectures, while the energy gain decreases. Indeed, for these results, we use random task placement which leads to long communications between tasks, and hence high Laser 
power to support these communications. This simulation context is the worst as possible, and we can imagine that a smart task placement should reduce these long communications and gives the opportunity to select more often the low Laser power levels. Nonetheless, these results demonstrate the efficiency of flexible wavelengths allocation and laser output power tuning to adapt the nanophotonic interconnects according to application requirements.

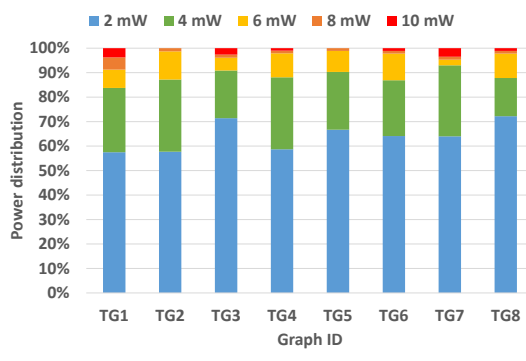

Figure 14: *

(a)

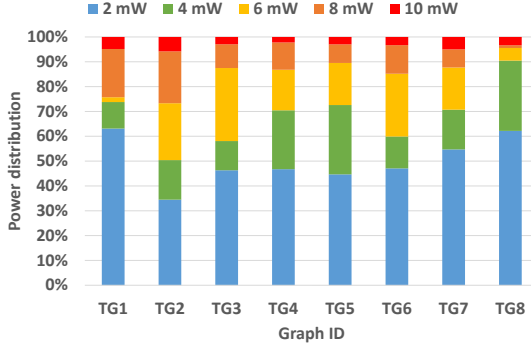

Figure 16: *

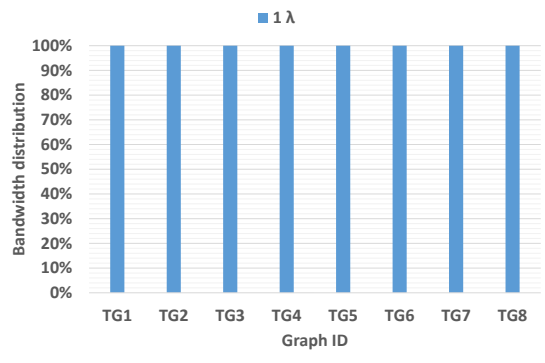

Figure 15: *

(b)

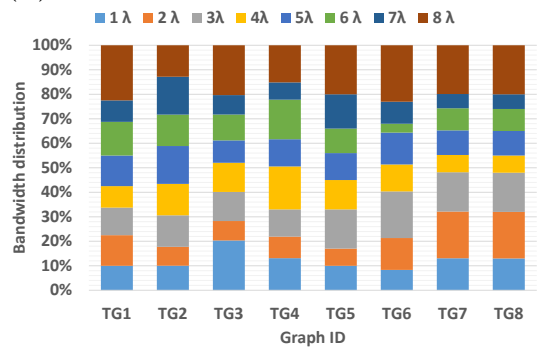

Figure 17: *

(c)

(d)

Figure 18: Distribution of laser power levels and allocated bandwidth for (a-b) low-power solutions and (c-d) high-performance solutions.

Figure 18(a) and 18(b) give the distribution of laser power levels and allocated bandwidth for the low-power solutions. On average, the $2 \mathrm{~mW}$ power level is selected in $60 \%$ of the cases and is followed by $4 \mathrm{~mW}$ ( $15 \%$ on average). Higher power levels are selected to compensate for the losses experienced by long range communications. Indeed, as shown in Figure 18(b), all the communications are allocated on a single wavelength for a minimum crosstalk effect. Figure 18(c) illustrates results for high-performance solutions. On average, $45 \%$ and $15 \%$ of the lasers are configured to $2 \mathrm{~mW}$ and $4 \mathrm{~mW}$ respectively. The lasers configured with a higher output power emit signals experiencing significant losses (i.e. long distance communications) or crosstalk. Figure 18(d) shows the number of wavelengths allocated distribution for each task graph for the same solutions. Taking TG8 as an example, $13 \%$ of the communications are carried out using a single 
wavelength, while $20 \%$ rely on all the wavelengths. Hence, the resulting ONoC configurations do not simply allocate all the wavelengths for each communication, even though maximum execution performance is reached. Indeed, our algorithm computes the maximum wavelengths to be allocated by considering simultaneous communications and computation time that may not be reduced.

These results demonstrate that the proposed approach to combine laser output power and wavelength allocation reaches the maximum execution performance while saving energy by using only the required optical resources. Furthermore, complex applications lead to solutions with very different energy and execution time figures. This confirms the opportunity induced by our off-line approach to adapt the $\mathrm{ONoC}$ configuration at run-time according to the execution context and QoS requirements.

\section{Conclusion}

Nanophotonic interconnects are promising solutions for high-performance and low-power on-chip communications. However, configuring an $\mathrm{ONoC}$ is challenging since the optimal use of optical resources depends on contradictory objectives related to BER requirements, expected execution performance and power budget. In this paper, we propose an off-line methodology allowing to simultaneously explore i) communication bandwidth allocation and ii) laser power levels. Inputs of the method are an application task graph, an architecture description and technological parameters. The resulting Pareto solutions include low-power solutions, which tend to minimize the number of used wavelengths, and high-performance solutions, for which multiple wavelengths are allocated to shorten the communication time. As an example, for a 63 -task application, the relative variation in the execution time and energy is $71 \%$ and $44 \%$ respectively. Solutions showing good energy-performance trade-offs are also found. Compared to baseline solutions for which laser power is fixed, our method leads to $74 \%$ energy reduction on average. The method also allows for the exploration $\mathrm{ONoC}$ design parameters such as the number of wavelengths, the number of waveguides, and the number of laser power levels.

\section{Acknowledgement}

This work has received a French Government support granted to the COMIN Labs excellence laboratory and managed by the National Research Agency in the "Investing for the Future" program under reference ANR-10-LABX-07-01. J. Luo is supported by China Scholarship Council (CSC).

\section{References}

[1] J. D. Owens, W. J. Dally, R. Ho, D. N. Jayasimha, S. W. Keckler, and L. S. Peh. Research challenges for on-chip interconnection networks. IEEE 
Micro, 27(5):96-108, Sept 2007.

[2] Alessandro Cilardo and Edoardo Fusella. Design automation for application-specific on-chip interconnects: A survey. Integration, the VLSI Journal, 52:102-121, 2016.

[3] Nir Magen, Avinoam Kolodny, Uri Weiser, and Nachum Shamir. Interconnect-power dissipation in a microprocessor. In Proceedings of the 2004 international workshop on System level interconnect prediction, pages 7-13. ACM, 2004.

[4] C. Batten, A. Joshi, V. Stojanovic, and K. Asanovic. Designing chip-level nanophotonic interconnection networks. IEEE Journal on Emerging and Selected Topics in Circuits and Systems, 2(2):137-153, June 2012.

[5] A. K. Dutta et al. WDM technologies: optical networks. Academic Press, 2004 .

[6] L. H. K. Duong et al. Coherent and incoherent crosstalk noise analyses in interchip/intrachip optical interconnection networks. IEEE Transactions on Very Large Scale Integration (VLSI) Systems, pages 2475-2487, July 2016.

[7] E. Fusella and A. Cilardo. Lighting up on-chip communications with photonics: Design tradeoffs for optical noc architectures. IEEE Circuits and Systems Magazine, 16(3):4-14, thirdquarter 2016.

[8] K. Deb et al. A fast elitist non-dominated sorting genetic algorithm for multi-objective optimization: Nsga-ii. In International Conference on Parallel Problem Solving From Nature, pages 849-858. Springer, 2000.

[9] H. Zang et al. A review of routing and wavelength assignment approaches for wavelength-routed optical wdm networks. Optical Networks Magazine, pages $47-60,2000$.

[10] TE Thomas and K Bala. Multiwavelength optical networks: a layered approach, 1999.

[11] S.V.R. Chittamuru et al. Improving crosstalk resilience with wavelength spacing in photonic crossbar-based network-on-chip architectures. In IEEE 58th International Midwest Symposium on Circuits and Systems (MWSCAS), pages 1-4, 2015.

[12] M. Nikdast et al. Crosstalk noise in wdm-based optical networks-on-chip: A formal study and comparison. IEEE Transactions on Very Large Scale Integration (VLSI) Systems, 23(11):2552-2565, 2015.

[13] X. Wang et al. Rpnoc: a ring-based packet-switched optical network-onchip. IEEE Photonics Technology Letters, 27(4):423-426, 2015. 
[14] S. Le Beux et al. Optical ring network-on-chip (ornoc): Architecture and design methodology. In Design, Automation \& Test in Europe, pages 1-6, 2011.

[15] E. Fusella et al. Phonocmap: an application mapping tool for photonic networks-on-chip. In 2016 Design, Automation \& Test in Europe Conference \& Exhibition (DATE), pages 289-292, 2016.

[16] L. Zhou and A. K. Kodi. Probe: Prediction-based optical bandwidth scaling for energy-efficient nocs. In 2013 Seventh IEEE/ACM International Symposium on Networks-on-Chip (NoCS), pages 1-8, April 2013.

[17] Roberto Rodes, Jesper Bevensee Jensen, Darko Zibar, Christian Neumeyr, Enno Rönneberg, Juergen Rosskopf, Markus Ortsiefer, and Idelfonso Tafur Monroy. Vertical-cavity surface-emitting laser based digital coherent detection for multigigabit long reach passive optical links. Microwave and Optical Technology Letters, 53(11):2462-2464, 2011.

[18] C. Sciancalepore, B. B. Bakir, X. Letartre, J. Harduin, N. Olivier, C. Seassal, J. M. Fedeli, and P. Viktorovitch. Cmos-compatible ultracompact 1.55 um emitting vcsels using double photonic crystal mirrors. IEEE Photonics Technology Letters, 24(6):455-457, March 2012.

[19] Enrico Macii et al. Ultra low-power electronics and design. Springer, 2004.

[20] Xiaowen Wu, Jiang Xu, Yaoyao Ye, Zhehui Wang, Mahdi Nikdast, and Xuan Wang. Suor: Sectioned undirectional optical ring for chip multiprocessor. ACM Journal on Emerging Technologies in Computing Systems (JETC), 10(4):29, 2014.

[21] Sébastien Le Beux, Hui Li, Ian O'Connor, Kazem Cheshmi, Xuchen Liu, Jelena Trajkovic, and Gabriela Nicolescu. Chameleon: Channel efficient optical network-on-chip. In Proceedings of the conference on Design, Automation \& Test in Europe, page 304. European Design and Automation Association, 2014.

[22] Abdullah Demir, Guowei Zhao, Sabine Freisem, Xiaohang Liu, and Dennis G Deppe. Scaling properties of lithographic vcsels. In SPIE OPTO, pages 79520O-79520O. International Society for Optics and Photonics, 2011.

[23] C. Li, R. Bai, A. Shafik, E. Z. Tabasy, G. Tang, C. Ma, C. H. Chen, Z. Peng, M. Fiorentino, P. Chiang, and S. Palermo. A ring-resonator-based silicon photonics transceiver with bias-based wavelength stabilization and adaptive-power-sensitivity receiver. In 2013 IEEE International Solid-State Circuits Conference Digest of Technical Papers, pages 124-125, Feb 2013.

[24] C. Chen, J. L. Abelln, and A. Joshi. Managing laser power in siliconphotonic noc through cache and noc reconfiguration. IEEE Transactions 
on Computer-Aided Design of Integrated Circuits and Systems, 34(6):972985, June 2015.

[25] C. Chen, T. Zhang, P. Contu, J. Klamkin, A. K. Coskun, and A. Joshi. Sharing and placement of on-chip laser sources in silicon-photonic nocs. In 2014 Eighth IEEE/ACM International Symposium on Networks-on-Chip (NoCS), pages 88-95, Sept 2014.

[26] F. Lan, R. Wu, C. Zhang, Y. Pan, and K. t. T. Cheng. Dlps: Dynamic laser power scaling for optical network-on-chip. In 2017 22nd Asia and South Pacific Design Automation Conference (ASP-DAC), pages 726-731, Jan 2017.

[27] M. Bahadori, S. Rumley, D. Nikolova, and K. Bergman. Comprehensive design space exploration of silicon photonic interconnects. Journal of Lightwave Technology, 34(12), June 2016.

[28] S. Xiao et al. Modeling and measurement of losses in silicon-on-insulator resonators and bends. Optics Express, 15(17):10553-10561, 2007.

[29] J. Luo, A. Elantably, V. D. Pham, C. Killian, D. Chillet, S. Le Beux, O. Sentieys, and I. O'Connor. Performance and energy aware wavelength allocation on ring-based wdm 3d optical noc. In Design, Automation Test in Europe Conference Exhibition (DATE), 2017, pages 1372-1377, March 2017.

[30] P. Dong, W. Qian, S. Liao, H. Liang, C. C. Kung, N. N. Feng, R. Shafiiha, J. Fong, D. Feng, A. V. Krishnamoorthy, and M. Asghari. Low loss silicon waveguides for application of optical interconnects. In IEEE Photonics

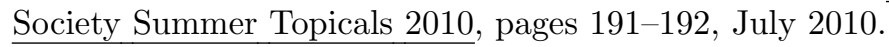

[31] M. Kennedy and A. K. Kodi. Laser pooling: Static and dynamic laser power allocation for on-chip optical interconnects. Journal of Lightwave Technology, 35(15):3159-3167, Aug 2017. 